\title{
One-hour glucose value as a long-term predictor of cardiovascular morbidity and mortality: the Malmö Preventive Project
}

\author{
Mette L Nielsen ${ }^{1}$, Manan Pareek ${ }^{1,2}$, Margrét Leósdóttir ${ }^{3}$, Karl-Fredrik Eriksson ${ }^{4}$, Peter M Nilsson $^{5}$ and \\ Michael H Olsen ${ }^{1,2}$
}

'Department of Endocrinology, Cardiovascular and Metabolic Preventive Clinic, Centre for Individualized Medicine in Arterial Diseases (CIMA), Odense University Hospital, Odense, Denmark, ${ }^{2}$ Cardiology Section, Department of Internal Medicine, Holbaek Hospital, Holbaek, Denmark, ${ }^{3}$ Department of Cardiology, Skåne University Hospital, Malmö, Sweden, ${ }^{4}$ Department of Clinical Sciences, Vascular Diseases, Lund University, Malmö, Sweden, and ${ }^{5}$ Department of Clinical Sciences, Lund University, Skåne University Hospital, Malmö, Sweden
Correspondence should be addressed to $M$ Lundgren Email mettelundgren@gmail.com

\begin{abstract}
Objective: To examine the predictive capability of a 1-h vs 2-h postload glucose value for cardiovascular morbidity and mortality.

Design: Prospective, population-based cohort study (Malmö Preventive Project) with subject inclusion 1974-1992. Methods: 4934 men without known diabetes and cardiovascular disease, who had blood glucose (BG) measured at 0 , $20,40,60,90$ and 120 min during an OGTT ( $30 \mathrm{~g}$ glucose per $\mathrm{m}^{2}$ body surface area), were followed for 27 years. Data on cardiovascular events and death were obtained through national and local registries. Predictive capabilities of fasting BG (FBG) and glucose values obtained during OGTT alone and added to a clinical prediction model comprising traditional cardiovascular risk factors were assessed using Harrell's concordance index (C-index) and integrated discrimination improvement (IDI).

Results: Median age was 48 (25th-75th percentile: 48-49) years and mean FBG $4.6 \pm 0.6 \mathrm{mmol} / \mathrm{L}$. FBG and 2-h postload BG did not independently predict cardiovascular events or death. Conversely, 1-h postload BG predicted cardiovascular morbidity and mortality and remained an independent predictor of cardiovascular death (HR: 1.09, 95\% Cl: 1.01-1.17, $P=0.02)$ and all-cause mortality (HR: $1.10,95 \% \mathrm{Cl}: 1.05-1.16, P<0.0001)$ after adjusting for various traditional risk factors. Clinical risk factors with added 1-h postload BG performed better than clinical risk factors alone, in predicting cardiovascular death (likelihood-ratio test, $P=0.02$ ) and all-cause mortality (likelihood-ratio test, $P=0.0001$; significant IDI, $P=0.0003$ ).

Conclusion: Among men without known diabetes, addition of 1-h BG, but not FBG or 2-h BG, to clinical risk factors provided incremental prognostic yield for prediction of cardiovascular death and all-cause mortality.
\end{abstract}

\section{Introduction}

The strong influence of type 2 diabetes on the risk of cardiovascular morbidity and mortality is undisputed $(1,2)$, and effective measures to predict and prevent both type 2 diabetes and related cardiovascular complications are desirable.
Fasting blood glucose (FBG) alone is not sufficient in predicting hyperglycemia-related mortality, while the standard 2-h postload glucose value enables the detection of subjects with impaired glucose tolerance (IGT), who have a significantly increased risk of death (3).

Published by Bioscientifica Ltd. 
Furthermore, in overweight subjects with IGT, intensive lifestyle intervention and pharmacological treatment can prevent progression into overt type 2 diabetes and possibly ameliorate the risk of related cardiovascular complications $(4,5,6)$. However, the 2-h OGTT has low sensitivity, has non-optimal specificity for incident diabetes and is timeconsuming, and thus it is considered to carry limited costeffectiveness and practicality (7). The past decade has seen several studies suggesting the superiority of a 1-h postload glucose value vs a 2 -h postload glucose value for the prediction of type 2 diabetes $(7,8,9,10,11,12,13,14)$. Previous data from the Malmö Preventive Project have also shown that individuals at high risk for future type 2 diabetes can be identified using multivariable prediction models that include both FBG and blood glucose (BG) obtained during OGTT, with a significantly better predictive capability achieved for shorter OGTT regimens compared with both FBG and the classical 2-h OGTT (15). This might extend to cardiovascular morbidity and hyperglycemia-related mortality. Therefore, the primary purposes of the present study were (1) to evaluate the prognostic value of FBG and BG values obtained at different time points during OGTT, in predicting cardiovascular morbidity and mortality, both isolated and in addition to a multivariable prediction model; (2) to assess whether BG measured at $60 \mathrm{~min}$ during OGTT $\left(\mathrm{BG}_{60}\right)$ provided better prognostic yield than FBG and BG measured at $120 \mathrm{~min}$ $\left(\mathrm{BG}_{120}\right)$ and (3) to assess whether the prognostic value of $\mathrm{BG}_{60}$ was influenced by glucose tolerance.

\section{Subjects and methods}

\section{Study population}

The Malmö Preventive Project (1974-1992, $n=33$ 346) was a population-based cohort study conducted among inhabitants in Malmö, Sweden, belonging to pre-specified birth cohorts between 1921 and 1949 (16). Progressively older subjects were recruited later during the inclusion process. In all, 22444 males and 10902 females attended the screening program, with an overall attendance rate of $71 \%$ ( $75 \%$ for men). All subjects answered a selfadministered questionnaire on lifestyle, family history of diabetes (first degree relatives) and cardiovascular disease, medical history and current medications. Current smoking was defined as smoking at least one cigarette daily, and sedentary lifestyle was defined as leisure time mostly spent on sedentary activities. Prevalent diabetes was defined as self-reported diabetes or according to the
1985 World Health Organization (WHO) criteria $(17,18)$. Prevalent cardiovascular disease was defined as selfreported cardiovascular disease (history of myocardial infarction (MI), stroke or transient ischemic attack) or according to national and local registries using the same diagnostic codes as for outcome (19). Height and weight in light indoor clothing were measured, from which body mass index (BMI) was calculated. Blood pressure was measured twice in the right arm after $10 \mathrm{~min}$ of supine rest, with the mean value recorded for analysis. Blood samples were obtained after $\geq 10$-h overnight fasting with measurement of $B G$, serum lipids and serum creatinine. BG levels were measured in capillary whole blood from fingertip samples and analyzed using the glucoseoxidase method (1974-1977) or the hexokinase method (1977-1992) (20). Serum samples were analyzed using the laboratory's standard methods. The Malmö Preventive Project was conducted in accordance with the Declaration of Helsinki.

\section{Outcome}

Follow-up time for each subject was defined as time elapsed from baseline screening to date of myocardial infarction, stroke, death, emigration or the last follow-up date up to 27 years. Cardiovascular events were recorded by national and local registries and comprised International Classification of Diseases (ICD-9) codes 410-414, 434, 430-438. Diagnosis of type 2 diabetes was recorded by 14 national and local registries and comprised International Classification of Diseases (ICD-10) codes E11.0-E11.9. The method to ascertain diagnosis of type 2 diabetes and cardiovascular events in the Swedish Inpatient Register has been previously validated (21). Positive predictive values were moderate for stroke and high for all other endpoints. Mortality follow-up was based on the national registry on causes of mortality at the Central Bureau of Statistics, Sweden.

\section{Final study population}

In participants without known diabetes, a 120-min OGTT was performed by ingestion of $30 \mathrm{~g}$ glucose per $\mathrm{m}^{2}$ body surface area (DuBois equation) in a $10 \%$ aqueous solution within $5 \mathrm{~min}$. BG levels were determined at $0,20,40,60$, 90 and $120 \mathrm{~min}$. Subjects without an OGTT with extra BG measurements ( $n=27728)$ were excluded from the present study, as were the 132 females of whom most did not have BG measured at the relevant intermediate time points. Remaining subjects with missing baseline variables $(n=70)$ 


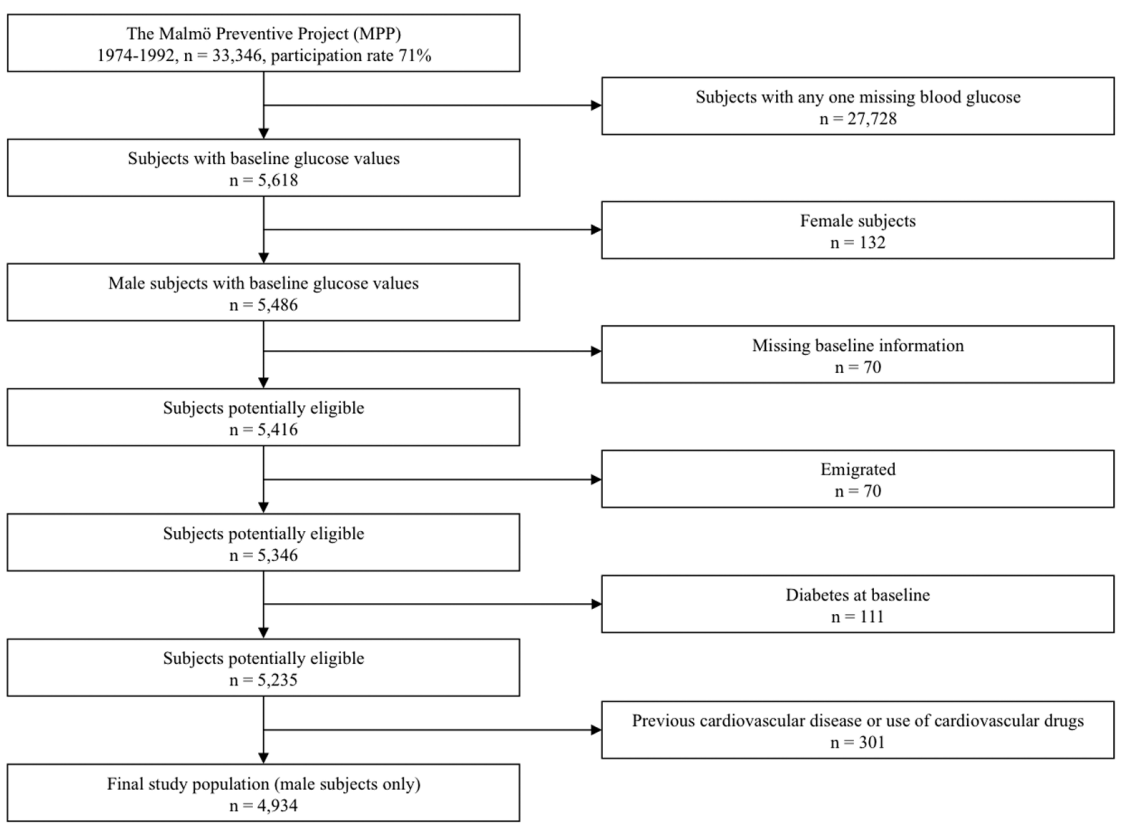

\section{Figure 1}

Flowchart showing the study population selection. or incomplete follow-up due to emigration $(n=70)$ were likewise excluded. Of the 5346 male subjects left, 111 had prevalent diabetes and 301 had previous cardiovascular disease or received cardiovascular medication (antihypertensives, antithrombotics, antiarrhythmics), leaving a final study population comprising 4934 males (Fig. 1). Of note, 315 subjects with IGT (defined locally as $\mathrm{BG}_{120} \geq 7.0 \mathrm{mmol} / \mathrm{L}$ ) who underwent lifestyle intervention (dietary advice and increased physical activity, most often including frequent visits at an outpatient clinic) for up to 12 years upon confirmation of their IGT status were not excluded (22).

\section{Statistical analysis}

Continuous variables were summarized by means and standard deviations (approximately normally distributed variables) or medians and 25th and 75th percentiles (nonnormally distributed variables). Categorical variables were presented by frequencies and corresponding percentages. Group-wise comparisons were performed using one-way analysis of variance (ANOVA), the Kruskal-Wallis test or Pearson's $\chi^{2}$-test, as appropriate. In order to define potential explanatory variables for the endpoints, i.e., incident myocardial infarction (nonfatal and fatal), stroke (nonfatal and fatal), death from cardiovascular causes and all-cause mortality, univariable Cox proportional-hazards regressions were applied on the following demographic and clinical variables: age, smoking status, BMI, systolic blood pressure, diastolic blood pressure, total cholesterol, triglycerides, creatinine, family history of diabetes and sedentary lifestyle. Since the distributions of serum triglycerides and creatinine were moderately positively skewed, they were both natural log-transformed for the regression analyses. Statistically significant and clinically relevant variables were included in the final multivariable Cox models, and stepwise backward elimination using the likelihood-ratio test was applied for adjustment of these models. The final regression model (clinical prediction model) included variables that were statistically significant in the multivariable models for at least one of the four endpoints. For BG, standardized values were calculated as the absolute value of $\mathrm{FBG}, \mathrm{BG}_{60}$ or $\mathrm{BG}_{120}$ for each subject divided by each of their standard deviations. Furthermore, we used interaction analyses to test whether glucose (in)tolerance influenced the prognostic value of $\mathrm{BG}_{60}$. This was done by including the interaction term for the continuous variable $\mathrm{BG}_{60}$ and the categorical variable, absence/presence of IGT, in the relevant regression models. The predictive abilities both alone and in addition to the clinical prediction model were tested with Harrell's concordance index (C-index) (23). The ability of each BG measurement during OGTT to enhance prognostication was also evaluated using integrated discrimination improvement (IDI) (24). Follow-up time was 27 years for all subjects without events, and supplementary analyses were done at shorter follow-up times (5, 10 and 20 years) to ensure that the results were independent of follow-up time. Analyses were focused on BG measured at 0,60 and $120 \mathrm{~min}$ during OGTT because of the overall 
better predictive capacity of $\mathrm{BG}_{60}$ compared with $\mathrm{BG}$ measured at 20 and $40 \mathrm{~min}$. BG measured at $90 \mathrm{~min}$ was comparable with $\mathrm{BG}_{60}$, but since this measurement required $30 \mathrm{~min}$ extra, it seemed reasonable to exclude it (15). The significance level was 0.05 (two-sided), and given the exploratory nature of this study, no adjustments for multiple comparisons were made. All analyses were carried out using IBM SPSS Statistics 23 (IBM) and Stata/ IC 14 (StataCorp LP).

\section{Results}

\section{Characteristics of the study population}

At baseline, median age was 48 (25th-75th percentile: $48-49)$ years, mean systolic blood pressure $130 \pm 15 \mathrm{mmHg}$ and mean BMI $24.8 \pm 3.2 \mathrm{~kg} / \mathrm{m}^{2}$. Total cholesterol was $5.8 \pm 1.0 \mathrm{mmol} / \mathrm{L}$. Subjects with IGT at baseline $(n=301)$ generally had a worse risk profile than their counterparts with NGT (Table 1). Similarly, a more adverse risk profile was present across $\mathrm{BG}_{60}$ tertiles (Table 1). During follow-up, 1381 composite cardiovascular events (myocardial infarction, stroke or cardiovascular death, whichever came first) were detected, corresponding to 12.3 cases per 1000 person-years. A total of 1517 individuals had died from any cause. IGT and the uppermost $\mathrm{BG}_{60}$ tertile appeared to be associated with poorer outcomes compared with NGT and the lower $\mathrm{BG}_{60}$ tertiles, respectively (Table 2 ).

\section{The clinical prediction model}

The following variables were statistically significant on univariable analysis for prediction of incident nonfatal plus fatal myocardial infarction and nonfatal plus fatal stroke: age, active smoking, BMI, systolic blood pressure, total cholesterol and triglycerides. Sedentary lifestyle and creatinine were significant on univariable analysis for prediction of cardiovascular death and all-cause mortality. The final Cox regression model (clinical prediction model) included age, active smoking, BMI, systolic blood pressure, total cholesterol, triglycerides and creatinine. This model performed better than anyone isolated BG measurement for the prediction of all endpoints, with C-index for the former being highest for cardiovascular death (C-index $=0.682$, 95\% confidence interval (CI): 0.66-0.70), followed by myocardial infarction (C-index $=0.666$, 95\% CI: 0.65-0.69), all-cause mortality (C-index $=0.638, \quad 95 \% \quad$ CI: $0.62-0.65$ ) and stroke (C-index $=0.612,95 \%$ CI: 0.59-0.63). 


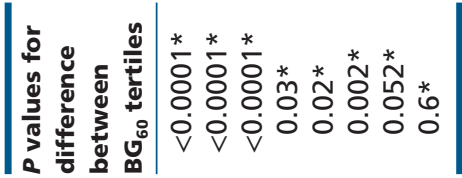

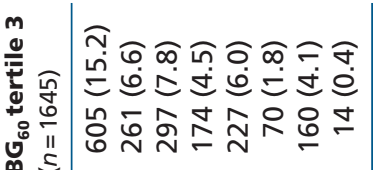

告

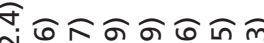

Єம் ปัฐ $\stackrel{m}{\sim}$ 등

เกลชิสล์ล

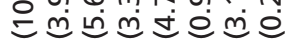

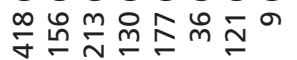

0

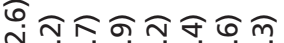
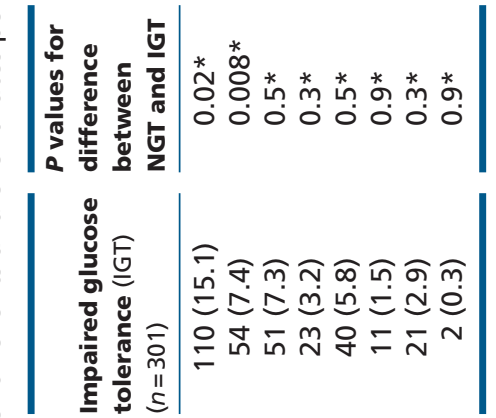

๒ம

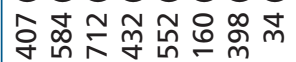

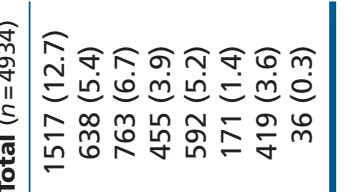

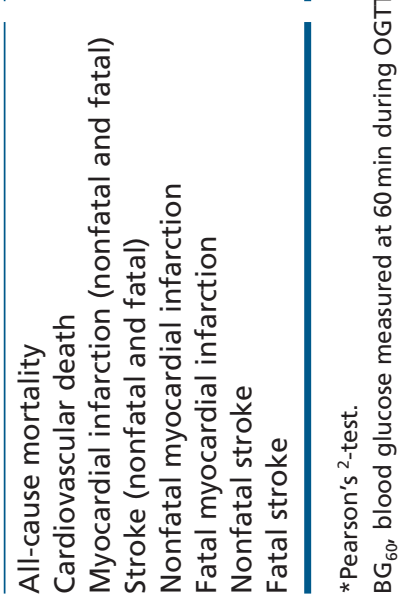

Fasting blood glucose and postload glucose values

At 27 years, $\mathrm{BG}_{60}$ alone was a significant predictor of fatal myocardial infarction (hazard ratio (HR): $1.22,95 \% \mathrm{CI}$ : 1.09-1.36, $P=0.0006)$, nonfatal myocardial infarction (HR: $1.10,95 \%$ CI: $1.02-1.18, P=0.01$ ), nonfatal plus fatal myocardial infarction (HR: 1.13, 95\% CI: 1.06-1.20, $P=0.0001$ ), nonfatal plus fatal stroke (HR: 1.14, 95\% CI: 1.06-1.24, $P=0.0008$ ), nonfatal stroke (HR: $1.14,95 \% \mathrm{CI}$ : 1.05-1.24, $P=0.002)$, death from cardiovascular causes (HR: $1.21,95 \%$ CI: $1.14-1.28, P<0.0001)$ and all-cause mortality (HR: $1.18,95 \%$ CI: 1.14-1.23, $P<0.0001$ ). BG $_{120}$ and FBG alone did not predict any of the endpoints (Table 3). At 20 years of follow-up, $\mathrm{BG}_{120}$ alone predicted cardiovascular death (HR: 1.13, 95\% CI: 1.02-1.25, $P=0.02)$, whereas FBG still did not predict any of the endpoints $\left(P \geq 0.21\right.$ for all), and $\mathrm{BG}_{60}$ remained superior to both $(P<0.0001)$

Fatal myocardial infarction was detected in 171 subjects, and fatal stroke was detected in 36 subjects. Accordingly, fatal myocardial infarction contributed the most to cardiovascular death. C-index for $\mathrm{BG}_{60}$ alone was consistently greater than that for FBG or $\mathrm{BG}_{120}$ (Table 4); however, significant comparisons were only observed for all-cause mortality, cardiovascular death, nonfatal plus fatal myocardial infarction and nonfatal plus fatal stroke ( $P \leq 0.02 ; P>0.05$ for the comparison with FBG (myocardial infarction) and for the comparison with $\mathrm{BG}_{120}$ (stroke)). The presence of IGT at baseline significantly interacted with the association between $\mathrm{BG}_{60}$ and all-cause mortality $(P=0.001)$, cardiovascular death $(P=0.0004)$ and nonfatal plus fatal stroke $(P=0.03)$, with higher predictive values of $\mathrm{BG}_{60}$ found among subjects with IGT at baseline (Fig. 2A, $\mathrm{B}, \mathrm{C}$ and $\mathrm{D})$. When $\mathrm{BG}_{60}$ and $\mathrm{BG}_{120}$ were both analyzed as continuous variables, $\mathrm{BG}_{120}$ modified the association between $\mathrm{BG}_{60}$ and myocardial infarction $(P=0.03)$.

The intervention, carried out in a minority of the study subjects, did not alter the estimates and did not interact with the association between any BG measurement and any endpoint $(P>0.05$ for all). Furthermore, incident type 2 diabetes during follow-up did not interact with any association between $\mathrm{BG}_{60}$ and the endpoints $(P \geq 0.50$ for all).

\section{Fasting blood glucose and oral glucose tolerance test in combination with the clinical prediction model}

After adjusting for the variables included in the clinical prediction model, neither $\mathrm{FBG}$ nor $\mathrm{BG}_{120}$ predicted any of the endpoints. However, $\mathrm{BG}_{60}$ remained independently 


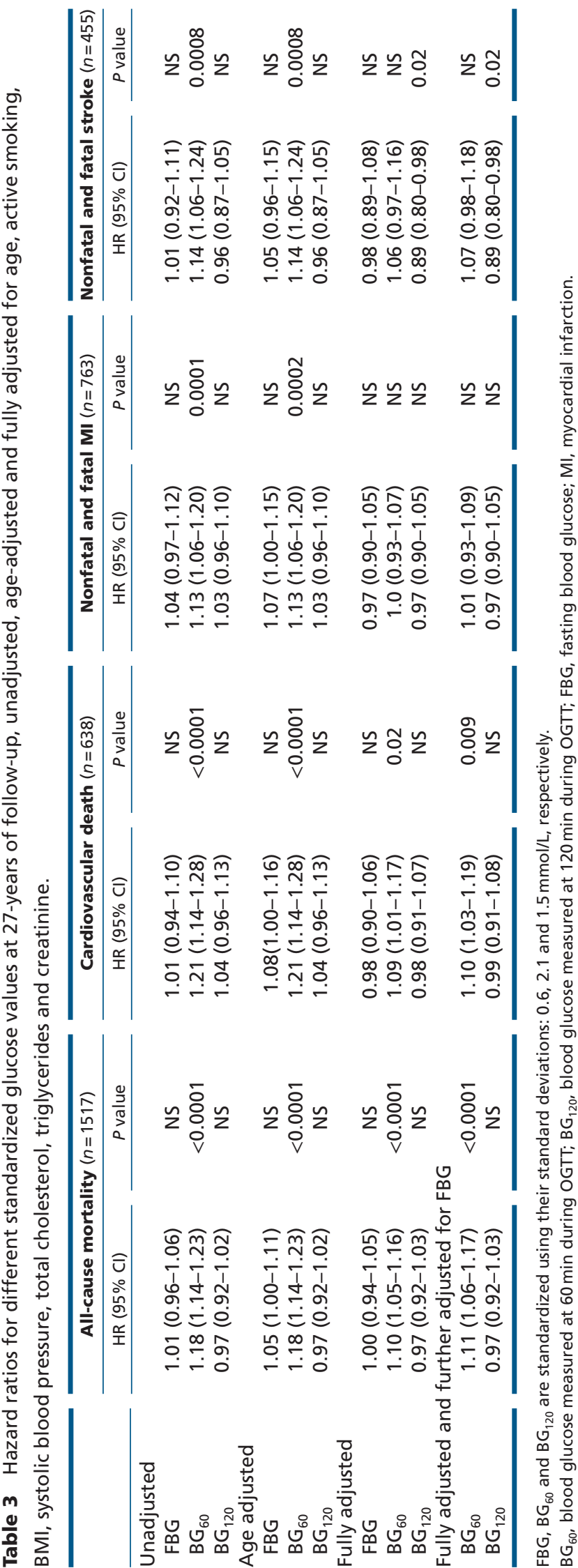

predictive of all-cause mortality (adjusted HR: 1.10, 95\% CI: $1.05-1.16, P<0.0001)$ and cardiovascular death (adjusted HR: 1.09, 95\% CI: 1.01-1.17, $P=0.02$ ) (Table 3). The predictive value of the fully adjusted $\mathrm{BG}_{60}$ model was also highest in subjects with IGT at baseline (Fig. 2A, B, C and $\mathrm{D}$ ). Adjusting for FBG did not reduce the predictive value of $\mathrm{BG}_{60}$ (Table 3).

Adding $\mathrm{BG}_{60}$ to the clinical prediction model significantly improved the prediction of all-cause mortality (likelihood-ratio test; $P=0.0001$ ) and cardiovascular death (likelihood-ratio test; $P=0.02$ ), although with no significant C-index increment. Addition of FBG or $\mathrm{BG}_{120}$ did not provide model improvement (likelihoodratio test; $P \geq 0.21$ for all). $\mathrm{BG}_{60}$ was further associated with significant IDI in predicting all-cause mortality $(P=0.0003)$. Conversely, $\mathrm{BG}_{120}$ was associated with a significant IDI in predicting stroke $(P=0.02)$, but the HR for $\mathrm{BG}_{120}$ was $<1$ in the fully adjusted prediction model.

The overall results did not change using shorter follow-up (Table 5).

\section{Discussion}

Our results show that among apparently healthy, middleaged men without known diabetes, BG measured at $60 \mathrm{~min}$ during OGTT provides better prognostic yield than both FBG and BG measured at $120 \mathrm{~min}$, in predicting long-term cardiovascular morbidity and mortality, both isolated and in addition to a clinical prediction model.

The conventional 2-h postload glucose value is known to be a significant, independent and better predictor of cardiovascular morbidity and mortality than fasting glucose $(3,25,26,27,28)$. We found a significant prediction of cardiovascular death with $\mathrm{BG}_{120}$, although only unadjusted, and no significant predictive value of FBG. However, the association between $\mathrm{BG}_{120}$ and cardiovascular death was only evident on supplementary analyses with a shorter follow-up time. The predictive capacity might have been diluted over time, given the old age, co-morbidity status and age-related events at the time of study completion. An additional explanation may be our relatively healthy (from a glucometabolic perspective) cohort, in which both fasting and 2-h glucose values generally were low and rarely reached the criteria for prediabetes. In contrast, other studies have largely evaluated persons with higher glucose values closer to or even fulfilling contemporary prediabetes criteria. The interventional program of lifestyle changes in most subjects with baseline IGT decreased mortality at 12 years 
Table 4 Harrell's C-indices for different glucose values at 27-years of follow-up alone and in addition to the clinical prediction model, which included age, active smoking, BMI, systolic blood pressure, total cholesterol, triglycerides and creatinine.

\begin{tabular}{|c|c|c|c|c|}
\hline & All-cause mortality & Cardiovascular death & Nonfatal and fatal MI & $\begin{array}{l}\text { Nonfatal and } \\
\text { fatal stroke }\end{array}$ \\
\hline \multicolumn{5}{|l|}{ Alone } \\
\hline FBG & 0.503 & 0.503 & 0.515 & 0.497 \\
\hline$B G_{60}$ & 0.557 & 0.567 & 0.543 & 0.546 \\
\hline $\mathrm{BG}_{120}$ & 0.515 & 0.503 & 0.506 & 0.516 \\
\hline$P^{a}$ & $<0.001$ & $<0.001^{a}$ & 0.06 & 0.02 \\
\hline$P^{\mathrm{b}}$ & 0.002 & $<0.001$ & 0.02 & 0.3 \\
\hline \multicolumn{5}{|c|}{ Together with the clinical prediction model } \\
\hline FBG & 0.638 & 0.682 & 0.667 & 0.612 \\
\hline $\mathrm{BG}_{60}$ & 0.641 & 0.685 & 0.666 & 0.613 \\
\hline $\mathrm{BG}_{120}$ & 0.638 & 0.682 & 0.666 & 0.616 \\
\hline $\mathrm{FBG}+\mathrm{BG}_{60}$ & 0.642 & 0.685 & 0.667 & 0.613 \\
\hline $\mathrm{FBG}+\mathrm{BG}_{120}$ & 0.638 & 0.682 & 0.666 & 0.616 \\
\hline $\mathrm{FBG}+\mathrm{BG}_{60}+\mathrm{BG}_{120}$ & 0.643 & 0.685 & 0.666 & 0.618 \\
\hline
\end{tabular}

$P$ values are for comparisons of Harrell's $C$-indices for $\mathrm{BG}_{60}$ vs Harrell's $\mathrm{C}$-indices for ${ }^{\mathrm{F}} \mathrm{FBG}$ and ${ }^{\mathrm{b} B \mathrm{~B}_{120}}$, respectively.

$\mathrm{BG}_{60}$, blood glucose measured at 60 min during OGTT; $\mathrm{BG}_{120}$, blood glucose measured at 120 min during OGTT; FBG, fasting blood glucose; MI, myocardial infarction.

(22), but there was no effect on long-term outcome (29). Furthermore, the local threshold for baseline IGT was rather low (7.0 rather than $7.8 \mathrm{mmol} / \mathrm{L})$. This is likely to have resulted in a rather large number of subjects at moderate-to-high risk being captured. Still, excluding all subjects with IGT would have lowered the variability in observed BG levels substantially and excluded a large number of individuals at high risk for clinical events.
Despite the above, $\mathrm{BG}_{60}$ significantly predicted myocardial infarction, stroke, cardiovascular death and all-cause mortality, and after adjustment for other cardiovascular risk factors, $\mathrm{BG}_{60}$ still predicted cardiovascular death and all-cause mortality. Lending support to our results, Orencia and coworkers (30) demonstrated that 1-h postload glucose was an independent risk factor for all-cause mortality at
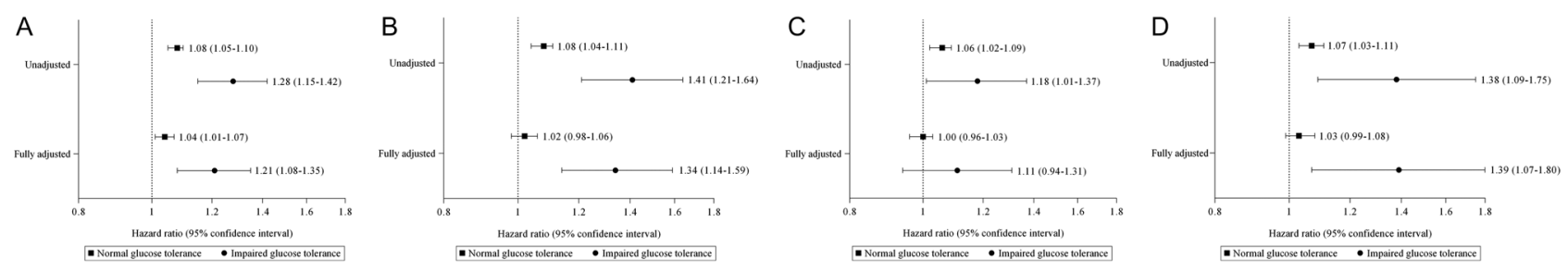

Figure 2

(A) Prediction of all-cause mortality by blood glucose obtained at 60-min OGTT in subjects with and without impaired glucose tolerance at baseline both unadjusted and fully adjusted for age, active smoking, BMI, systolic blood pressure, total cholesterol, triglycerides and creatinine. Hazard ratios are calculated for absolute, unstandardized glucose values. NGT, normal glucose tolerance; IGT, impaired glucose tolerance. (B) Prediction of cardiovascular mortality by blood glucose obtained at 60 min during OGTT in subjects with and without impaired glucose tolerance at baseline both unadjusted and fully adjusted for age, active smoking, BMI, systolic blood pressure, total cholesterol, triglycerides and creatinine. Hazard ratios are calculated for absolute, unstandardized glucose values. NGT, normal glucose tolerance; IGT, impaired glucose tolerance. (C). Prediction of myocardial infarction (nonfatal + fatal) by blood glucose obtained at 60 min during OGTT test in subjects with and without impaired glucose tolerance at baseline both unadjusted and fully adjusted for age, active smoking, BMI, systolic blood pressure, total cholesterol, triglycerides and creatinine. Hazard ratios are calculated for absolute, unstandardized glucose values. NGT, normal glucose tolerance; IGT, impaired glucose tolerance. (D) Prediction of stroke (nonfatal + fatal) by blood glucose obtained at 60 min during OGTT in subjects with and without impaired glucose tolerance at baseline both unadjusted and fully adjusted for age, active smoking, BMI, systolic blood pressure, total cholesterol, triglycerides and creatinine. Hazard ratios are calculated for absolute, unstandardized glucose values. NGT, normal glucose tolerance; IGT, impaired glucose tolerance. 


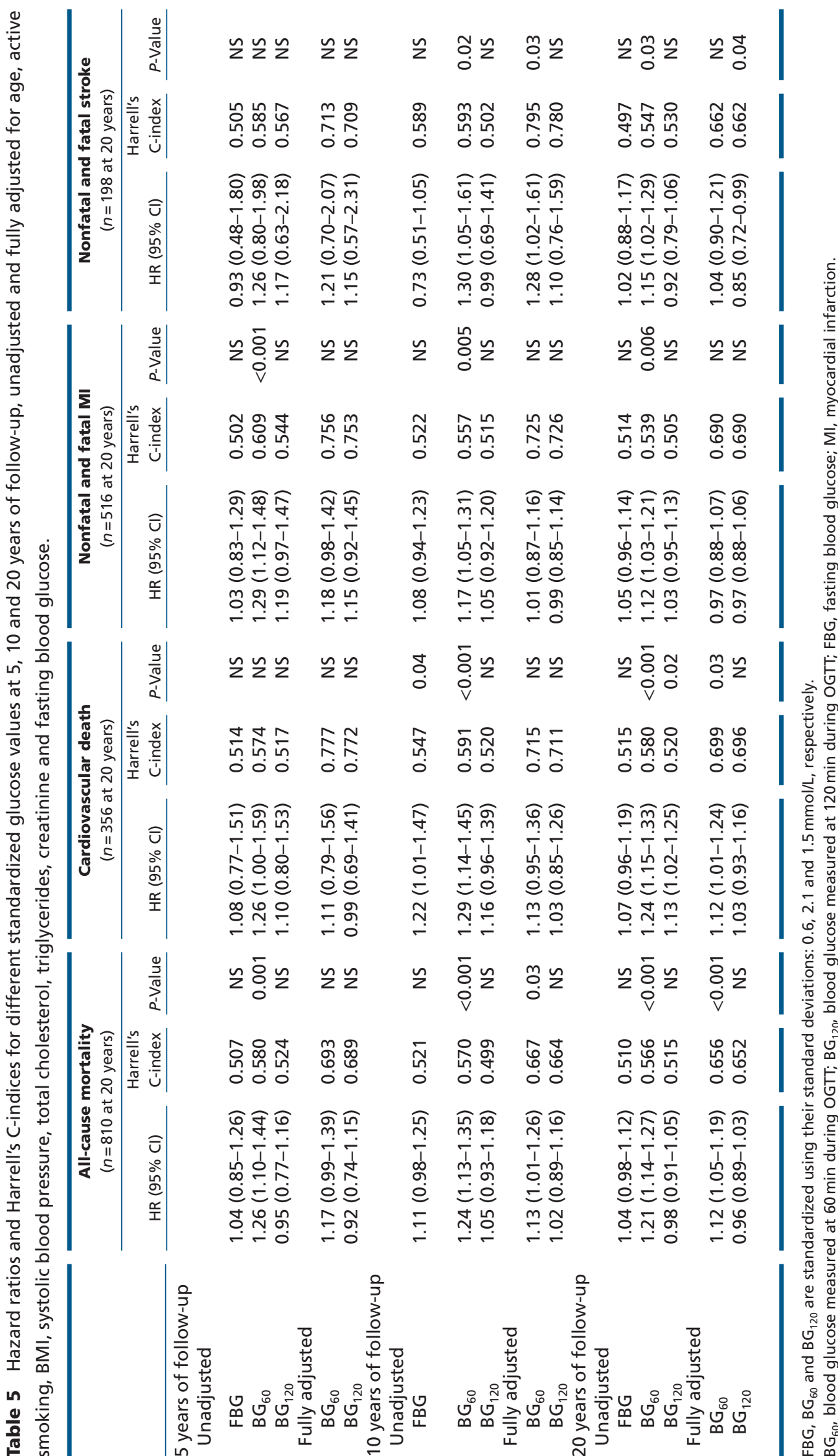


long-term (22 years) follow-up in middle-aged men without diabetes. Higher postload glucose levels were also independent risk factors for the major cardiovascular diseases. However, there was no direct comparison with fasting or 2-h glucose measurements. This was also the case for a 44-year prospective study, in which a strong and graded relationship was found between 1-h glucose quartiles and both total and cardiovascular mortality, independently of traditional cardiovascular risk factors, in men without diabetes (31). In a third study by Meisinger and coworkers (32), it was likewise established that a higher 1-h postload glucose value was an independent long-term (up to 30 years) predictor of all-cause mortality. However, the investigators used a very high partition value $(200 \mathrm{mg} / \mathrm{dL}=11.1 \mathrm{mmol} / \mathrm{L})$ and did not provide a comparison with fasting or 2-h glucose values. Data from the Finnish Diabetes Prevention Study showed that among persons with IGT, both 1-h and 2-h plasma glucose values and their temporal patterns were significantly associated with cardiovascular events (myocardial infarction, stroke, unstable angina, coronary artery bypass graft or percutaneous coronary intervention), although only the 2-h glucose remained significant in pairwise comparisons (33). Concerning reclassification abilities, Bergman and coworkers ( 7 ) found that a 1 -h glucose value $>155 \mathrm{mg} / \mathrm{dL}$ $(>8.6 \mathrm{mmol} / \mathrm{L})$ predicted mortality among individuals in whom the 2 -h level was $<140 \mathrm{mg} / \mathrm{dL}(<7.8 \mathrm{mmol} / \mathrm{L})$. Finally, an elaborate study by Hulman and coworkers identified four distinct glucose patterns during an OGTT with glucose measurements at 0, 30 and $120 \mathrm{~min}$. Among these healthy participants without diabetes at baseline, the risks of incident diabetes and of all-cause mortality were significantly increased with an elevated glucose level at $30 \mathrm{~min}$, risks that were independent of glucose levels at 0 and $120 \mathrm{~min}$ (34). The same group of authors was also involved in another study of glucose response curves during OGTT, in which they observed a variety of responses despite similar fasting and 2-h glucose values. These responses differed with respect to their associations with cardiovascular and metabolic risk factors. This in and of itself suggests the usefulness of an intermediate, i.e., between 0 and $120 \mathrm{~min}$, measurement of postload glucose (35).

\section{Influence of impaired glucose tolerance at baseline}

The interactions with IGT and the association between $\mathrm{BG}_{60}$ and all-cause mortality, cardiovascular death and stroke, may suggest that elevated $\mathrm{BG}_{60}$ does not merely precede elevated $\mathrm{BG}_{120}$, as a smaller effect of increased
$\mathrm{BG}_{60}$ in subjects with increased $\mathrm{BG}_{120}$ would then be expected. Possibly, having both abnormal $\mathrm{BG}_{60}$ and $\mathrm{BG}_{120}$ increases the possibility of correct categorization. Still, the interactions for the continuous BG variables suggest other explanations. One very interesting hypothesis could be that high $\mathrm{BG}_{60}$ is particularly harmful, from a cardiovascular perspective, if allowed to stay elevated for at least 60 additional minutes. Accordingly, these interactions may reflect a dissociation between prediction of type 2 diabetes and cardiovascular events, as both $\mathrm{BG}_{60}$ and $\mathrm{BG}_{120}$ appear to be markers of prediabetes, while the mechanisms by which $\mathrm{BG}_{60}$ and $\mathrm{BG}_{120}$ predict cardiovascular events seemingly differ (33). Finally, it must be emphasized that the intervention program of lifestyle changes may have influenced the results, although given the interaction between $\mathrm{BG}_{60}$ and $\mathrm{BG}_{120}$, this does not seem to be the case at long-term follow-up.

\section{Postload glucose values and clinical risk factors}

Addition of $\mathrm{BG}_{60}$ to traditional cardiovascular risk factors significantly improved the predictive capability, and there was also significant IDI in the prediction of allcause mortality, when adding $\mathrm{BG}_{60}$ to these risk factors. Conversely, Stern and coworkers (36) did not find a significant model improvement after addition of the standard 2-h postload glucose measurement to a clinical prediction model. The investigators thus suggested that prediction models should only include readily available clinical variables. Obviously, this is attractive because of the ease of administration, convenience, acceptability to screened subjects and lower cost. However, since the burden of type 2 diabetes and complications is rapidly rising, we believe that subjects at high risk of developing IGT and type 2 diabetes could be identified earlier using $\mathrm{BG}_{60}$. It is very likely that in subjects with an abnormal $\mathrm{BG}_{60}$, lifestyle intervention may prevent progression to IGT, type 2 diabetes, cardiovascular events and ultimately death.

\section{Influence of development of type 2 diabetes during follow-up}

Another question that appears is whether such elevated cardiovascular risk is restricted to individuals who develop diabetes during follow-up, but in the present study, incident type 2 diabetes did not significantly interact with the association between $\mathrm{BG}_{60}$ and incident cardiovascular events or death. Only few studies have explored this because of the absence of follow-up data 
on diabetes diagnosis; however, in accordance with our findings, Qiao and coworkers (37) demonstrated that baseline IGT, although defined by the standard 2-h OGTT, was an independent predictor for 10-year cardiovascular morbidity and mortality as well as total mortality, not confounded by the subsequent development of diabetes.

\section{Limitations}

A comparison between $\mathrm{HbA}_{1 \mathrm{c}}$ and glucose values obtained during OGTT would have been desirable, but such measurements were not done. OGTT was performed only at baseline and therefore subjected to regression dilution bias (38). Furthermore, at the time of inclusion, a standard OGTT comprised a $30 \mathrm{~g} / \mathrm{m}^{2}$ glucose load which, however, seems to yield results similar to the standard $75 \mathrm{~g}$ load (39). Our exclusion of a significant proportion of the original study population, although based on financial reasons (lack of extra BG measurements), constitutes a major limitation and prevents us from extending our results beyond middle-aged white men. Additionally, the lifestyle intervention performed in subjects with IGT at baseline may potentially have influenced the predictive capability of the 2-h glucose measurement, even though the follow-up data and analyses did not indicate this in neither the present nor in a previous study (29). In fact, excluding these subjects at the highest likelihood of developing type 2 diabetes and as such, the outcomes examined in this study, might have underestimated the true risk of complications. Despite significant differences in several comparisons between the prediction models, the discriminative values and their numerical differences were of limited magnitude. This may suggest limited clinical relevance. However, follow-up time was very long, which attenuated the discriminative ability of all models as previously shown (15). Furthermore, it should be remembered that prediction models generally tend to overestimate the actual event risk when applied to the population from which they were derived (40). Lastly, different between-study results might further be due to variations in sex ratio, ethnicity, sample sizes, inclusion criteria, co-variables included, glucose load used for OGTT, follow-up duration and definition of outcome variables.

\section{Clinical perspectives}

Shorter examination time is associated with lower cost and greater convenience for both patients and health care professionals. Although the introduction of $\mathrm{HbA} 1_{c}$ as a diagnostic tool for diabetes has led to performance of fewer OGTTs, it is well known that these methods display limited concordance (41). Therefore, a role for OGTT likely exists in subjects with a high normal or an only slightly elevated $\mathrm{HbA}_{\mathrm{c}}$, in order to reach a definitive diagnostic decision. With an increasing number of studies demonstrating a strong superior capacity compared with FBG and the 2-h glucose value in identifying subjects with a high risk of developing type 2 diabetes and related complications, it seems reasonable to consider the 1-h glucose value in order to benefit from early prevention in subjects with high risk of future type 2 diabetes and cardiovascular complications. Still, our findings need replication and external validation. In addition, interventional studies are necessary to prove the benefit of secondary prevention in high-risk individuals found through non-traditional methods.

In conclusion, in this prospective population-based cohort study, we found an OGTT-derived 60-min BG provided better prognostic value than FBG or 120-min postload BG, in predicting long-term cardiovascular morbidity and mortality, in apparently healthy, middleaged, men without known diabetes.

\section{Declaration of interest}

The authors declare that there is no conflict of interest that could be perceived as prejudicing the impartiality of this study.

\section{Funding}

This study was funded by a research grant from The Heart and Lung Foundation of Sweden and The Danish Diabetes Academy supported by the Novo Nordisk Foundation.

\section{Author contribution statement}

Mette Nielsen contributed substantially to the conception of the hypothesis, the design of the work, analysis of the work, interpretation of data for the work, drafting the work, critical revision for important intellectual content, final approval of the version to be published and agrees to be accountable for all aspects of the work in ensuring that questions related to the accuracy or integrity of any part of the work are appropriately investigated and resolved. Manan Pareek contributed substantially to the conception of the hypothesis, the design of the work, analysis of the work, critical revision for important intellectual content, final approval of the version to be published and agrees to be accountable for all aspects of the work in ensuring that questions related to the accuracy or integrity of any part of the work are appropriately investigated and resolved. Margrét Leósdóttir, Karl-Fredrik Eriksson and Peter M Nilsson contributed substantially to the acquisition of data for the work, critical revision for important intellectual content, the final approval of the version to be published and agrees to be accountable for all aspects of the work in ensuring that questions related to the accuracy or integrity of any part of the work are appropriately investigated and resolved. Michael Olsen contributed substantially to the conception of the hypothesis, the design of the work, analysis of the work, interpretation of data for the work, drafting the work, critical revision for important intellectual content, final approval of the version to be published and 
agrees to be accountable for all aspects of the work in ensuring that questions related to the accuracy or integrity of any part of the work are appropriately investigated and resolved.

\section{References}

1 Kannel WB \& McGee DL. Diabetes and cardiovascular disease. The Framingham study. JAMA 1979241 2035-2038. (https://doi. org/10.1001/jama.1979.03290450033020)

2 Stamler J, Vaccaro O, Neaton JD \& Wentworth D. Diabetes, other risk factors, and 12-year cardiovascular mortality for men screened in the Multiple Risk Factor Intervention Trial. Diabetes Care 199316 434-444. (https://doi.org/10.2337/diacare.16.2.434)

3 Glucose tolerance and mortality: comparison of WHO and American Diabetes Association diagnostic criteria. The DECODE study group. European Diabetes Epidemiology Group. Diabetes epidemiology: collaborative analysis of diagnostic criteria in Europe. Lancet 1999 354 617-621.

4 Knowler WC, Barrett-Connor E, Fowler SE, Hamman RF, Lachin JM, Walker EA, Nathan DM \& Diabetes Prevention Program Research Group. Reduction in the incidence of type 2 diabetes with lifestyle intervention or metformin. New England Journal of Medicine 2002346 393-403. (https://doi.org/10.1056/NEJMoa012512)

5 Tuomilehto J, Lindstrom J, Eriksson JG, Valle TT, Hamalainen H, Ilanne-Parikka P, Keinänen-Kiukaanniemi S, Laakso M, Louheranta A, Rastas M, et al. Prevention of type 2 diabetes mellitus by changes in lifestyle among subjects with impaired glucose tolerance. New England Journal of Medicine 2001344 1343-1350. (https://doi. org/10.1056/NEJM200105033441801)

6 Chiasson JL, Josse RG, Gomis R, Hanefeld M, Karasik A, Laakso M \& STOP-NIDDM Trail Research Group. Acarbose for prevention of type 2 diabetes mellitus: the STOP-NIDDM randomised trial. Lancet 2002 359 2072-2077. (https://doi.org/10.1016/S0140-6736(02)08905-5)

7 Bergman M, Chetrit A, Roth J \& Dankner R. One-hour postload plasma glucose level during the OGTT predicts mortality: observations from the Israel Study of Glucose Intolerance, Obesity and Hypertension. Diabetic Medicine 201633 1060-1066.

8 Abdul-Ghani MA, Williams K, DeFronzo RA \& Stern M. What is the best predictor of future type 2 diabetes? Diabetes Care 200730 1544-1548. (https://doi.org/10.2337/dc06-1331)

9 Dankner R, Abdul-Ghani MA, Gerber Y, Chetrit A, Wainstein J $\&$ Raz I. Predicting the 20-year diabetes incidence rate. Diabetes/ Metabolism Research and Reviews 200723 551-558. (https://doi. org/10.1002/dmrr.728)

10 Abdul-Ghani MA, Abdul-Ghani T, Ali N \& Defronzo RA. One-hour plasma glucose concentration and the metabolic syndrome identify subjects at high risk for future type 2 diabetes. Diabetes Care 200831 1650-1655. (https://doi.org/10.2337/dc08-0225)

11 Abdul-Ghani MA, Lyssenko V, Tuomi T, DeFronzo RA \& Groop L. Fasting versus postload plasma glucose concentration and the risk for future type 2 diabetes: results from the Botnia Study. Diabetes Care 200932 281-286. (https://doi.org/10.2337/dc08-1264)

12 Priya M, Anjana RM, Chiwanga FS, Gokulakrishnan K, Deepa M \& Mohan V. 1-hour venous plasma glucose and incident prediabetes and diabetes in Asian Indians. Diabetes Technology and Therapeutics 201315 497-502. (https://doi.org/10.1089/dia.2013.0025)

13 Alyass A, Almgren P, Akerlund M, Dushoff J, Isomaa B, Nilsson P, Tuomi T, Lyssenko V, Groop L \& Meyre D. Modelling of OGTT curve identifies $1 \mathrm{~h}$ plasma glucose level as a strong predictor of incident type 2 diabetes: results from two prospective cohorts. Diabetologia 201558 87-97. (https://doi.org/10.1007/s00125-014-3390-x)

14 Fiorentino TV, Marini MA, Andreozzi F, Arturi F, Succurro E, Perticone M, Sciacqua A, Hribal ML, Perticone F \& Sesti G. One-hour postload hyperglycemia is a stronger predictor of type 2 diabetes than impaired fasting glucose. Journal of Clinical Endocrinology and
Metabolism 2015100 3744-3751. (https://doi.org/10.1210/jc.20152573)

15 Nielsen ML, Pareek M, Leosdottir M, Hojlund K, Eriksson KF, Nilsson P \& Olsen MH. Follow-up duration influences the relative importance of OGTT and optimal timing of glucose measurements for predicting future type 2 diabetes. European Journal of Endocrinology 2016174 591-600. (https://doi.org/10.1530/EJE-15-1221)

16 Berglund G, Eriksson KF, Israelsson B, Kjellstrom T, Lindgarde F, Mattiasson I, Nilsson JA \& Stavenow L. Cardiovascular risk groups and mortality in an urban Swedish male population: the Malmo Preventive Project. Journal of Internal Medicine 1996239 489-497. (https://doi.org/10.1046/j.1365-2796.1996.483819000.x)

17 Diabetes mellitus. Report of a WHO Study Group. World Health Organization Technical Report Series 1985727 1-113.

18 Eriksson KF \& Lindgarde F. Impaired glucose tolerance in a middleaged male urban population: a new approach for identifying highrisk cases. Diabetologia 199033 526-531. (https://doi.org/10.1007/ BF00404139)

19 Nilsson P, Nilsson JA, Hedblad B, Eriksson KF \& Berglund G. Hyperinsulinaemia as long-term predictor of death and ischaemic heart disease in nondiabetic men: the Malmo Preventive Project. Journal of Internal Medicine 2003253 136-145. (https://doi. org/10.1046/j.1365-2796.2003.01064.x)

20 Carroll JJ, Smith N \& Babson AL. A colorimetric serum glucose determination using hexokinase and glucose-6-phosphate dehydrogenase. Biochemical Medicine 19704 171-180. (https://doi. org/10.1016/0006-2944(70)90093-1)

21 Ludvigsson JF, Andersson E, Ekbom A, Feychting M, Kim JL, Reuterwall C, Heurgren M \& Olausson PO. External review and validation of the Swedish national inpatient register. BMC Public Health 201111 450. (https://doi.org/10.1186/1471-2458-11-450)

22 Eriksson KF \& Lindgarde F. No excess 12 -year mortality in men with impaired glucose tolerance who participated in the Malmo Preventive Trial with diet and exercise. Diabetologia 199841 1010-1016. (https://doi.org/10.1007/s001250051024)

23 Harrell FE Jr, Califf RM, Pryor DB, Lee KL \& Rosati RA. Evaluating the yield of medical tests. JAMA 1982247 2543-2546. (https://doi. org/10.1001/jama.1982.03320430047030)

24 Pencina MJ, D'Agostino RB Sr, D'Agostino RB Jr \& Vasan RS Evaluating the added predictive ability of a new marker: from area under the ROC curve to reclassification and beyond. Statistics in Medicine 200827 157-172; discussion 207-212. (https://doi. org/10.1002/sim.2929)

25 Ford ES, Zhao G \& Li C. Pre-diabetes and the risk for cardiovascular disease: a systematic review of the evidence. Journal of the American College of Cardiology 201055 1310-1317. (https://doi.org/10.1016/j. jacc.2009.10.060)

26 Decode Study Group EDEG. Is the current definition for diabetes relevant to mortality risk from all causes and cardiovascular and noncardiovascular diseases? Diabetes Care 200326 688-696.

27 de Vegt F, Dekker JM, Ruhe HG, Stehouwer CD, Nijpels G, Bouter LM $\&$ Heine RJ. Hyperglycaemia is associated with all-cause and cardiovascular mortality in the Hoorn population: the Hoorn Study. Diabetologia 199942 926-931. (https://doi.org/10.1007/ s001250051249)

28 Meigs JB, Nathan DM, D’Agostino RB Sr, Wilson PW \& Framingham Offspring S. Fasting and postchallenge glycemia and cardiovascular disease risk: the Framingham Offspring Study. Diabetes Care 200225 1845-1850. (https://doi.org/10.2337/diacare.25.10.1845)

29 Berglund G, Nilsson P, Eriksson KF, Nilsson JA, Hedblad B, Kristenson H \& Lindgärde F. Long-term outcome of the Malmo preventive project: mortality and cardiovascular morbidity. Journal of Internal Medicine 2000247 19-29. (https://doi.org/10.1046/j.13652796.2000.00568.x)

30 Orencia AJ, Daviglus ML, Dyer AR, Walsh M, Greenland P \& Stamler J. One-hour postload plasma glucose and risks of fatal 
coronary heart disease and stroke among nondiabetic men and women: the Chicago Heart Association Detection Project in Industry (CHA) Study. Journal of Clinical Epidemiology 199750 1369-1376. (https://doi.org/10.1016/S0895-4356(97)00201-1)

31 Strandberg TE, Pienimaki T, Strandberg AY, Salomaa VV, Pitkala KH, Tilvis RS \& Miettinen TA. One-hour glucose, mortality, and risk of diabetes: a 44-year prospective study in men. Archives of Internal Medicine 2011171 941-943. (https://doi.org/10.1001/ archinternmed.2011.183)

32 Meisinger C, Wolke G, Brasche S, Strube G \& Heinrich J. Postload plasma glucose and 30-year mortality among nondiabetic middleaged men from the general population: the ERFORT Study. Annals of Epidemiology 200616 534-539. (https://doi.org/10.1016/j. annepidem.2005.10.008)

33 Lind M, Tuomilehto J, Uusitupa M, Nerman O, Eriksson J, IlanneParikka P, Keinänen-Kiukaanniemi S, Peltonen M, Pivodic A \& Lindström J. The association between HbA1c, fasting glucose, 1-hour glucose and 2-hour glucose during an oral glucose tolerance test and cardiovascular disease in individuals with elevated risk for diabetes. PLoS ONE 20149 e109506. (https://doi.org/10.1371/journal. pone.0109506)

34 Hulman A, Vistisen D, Glumer C, Bergman M, Witte DR \& Faerch K. Glucose patterns during an oral glucose tolerance test and associations with future diabetes, cardiovascular disease and all-cause mortality rate. Diabetologia 201861 101-107. (https://doi. org/10.1007/s00125-017-4468-z)

35 Hulman A, Simmons RK, Vistisen D, Tabak AG, Dekker JM, Alssema M, Rutters F, Koopman AD, Solomon TP, Kirwan JP, et al. Heterogeneity in glucose response curves during an oral glucose tolerance test and associated cardiometabolic risk. Endocrine $2017 \mathbf{5 5}$ 427-434. (https://doi.org/10.1007/s12020-016-1126-z)

36 Stern MP, Fatehi P, Williams K \& Haffner SM. Predicting future cardiovascular disease: do we need the oral glucose tolerance test? Diabetes Care 200225 1851-1856. (https://doi.org/10.2337/ diacare.25.10.1851)

37 Qiao Q, Jousilahti P, Eriksson J \& Tuomilehto J. Predictive properties of impaired glucose tolerance for cardiovascular risk are not explained by the development of overt diabetes during follow-up. Diabetes Care 200326 2910-2914. (https://doi.org/10.2337/diacare.26.10.2910)

38 Clarke R, Shipley M, Lewington S, Youngman L, Collins R, Marmot M \& Peto R. Underestimation of risk associations due to regression dilution in long-term follow-up of prospective studies. American Journal of Epidemiology 1999150 341-353. (https://doi. org/10.1093/oxfordjournals.aje.a010013)

39 Eriksson KF \& Lindgarde F. Prevention of type 2 (non-insulindependent) diabetes mellitus by diet and physical exercise. The 6-year Malmo feasibility study. Diabetologia 199134 891-898. (https://doi.org/10.1007/BF00400196)

40 Abbasi A, Peelen LM, Corpeleijn E, van der Schouw YT, Stolk RP, Spijkerman AM, van der A DL, Moons KG, Navis G, Bakker SJ, et al. Prediction models for risk of developing type 2 diabetes: systematic literature search and independent external validation study. $B M J$ 2012345 e5900. (https://doi.org/10.1136/bmj.e5900)

41 Collaboration NCDRF. Effects of diabetes definition on global surveillance of diabetes prevalence and diagnosis: a pooled analysis of 96 population-based studies with 331288 participants. Lancet Diabetes and Endocrinology 20153 624-637. (https://doi.org/10.1016/ S2213-8587(15)00129-1)

Received 8 October 2017

Revised version received 15 December 2017

Accepted 19 December 2017 